\title{
New topological measures on the torus
}

\author{
by
}

\author{
Finn F. Knudsen (Trondheim)
}

\begin{abstract}
Recently Entov and Polterovich [1] asked if the Grubb measure was the only symplectic topological measure on the torus. Much to our surprise we discovered a whole new class of intrinsic simple topological measures on the torus, many of which were symplectic.
\end{abstract}

Introduction. Topological measures were first introduced by Johan Aarnes under the name of quasi-measures. Associated with such a measure there is also a theory of integration for continuous functions. The existence of a non-subadditive quasi-measure proved the existence of a non-linear quasi-state on a commutative $C^{*}$-algebra. See [5]. The definition given by Aarnes is the following.

Definition 1. A topological measure on a compact Hausdorff space $X$ is a non-negative function $\mu$, defined on the collection of open or closed subsets of $X, \mathcal{A}(X)$, with the following properties.

(a) $\mu(\emptyset)=0$.

(b) If $A_{1} \subseteq A_{2}$, then $\mu\left(A_{1}\right) \leq \mu\left(A_{2}\right)$.

(c) If $A=\bigcup_{i=1}^{n} A_{i}$ is a disjoint union, then $\mu(A)=\sum_{i=1}^{n} \mu\left(A_{i}\right)$.

(d) If $U$ is open, then $\mu(U)=\sup \{\mu(C) \mid C \subseteq U$ and $C$ closed $\}$.

A topological measure is called simple if it takes only values 0 and 1 .

A topological measure $\mu$ on the torus is determined by its components $\mu_{\varepsilon}$ on equivalence classes of 2 -sided sets, and $\mu_{c}$ on the 1-sided sets. See [2]. If $\mu_{c}(A)=0$ on all contractible sets $A$, the measure is called intrinsic. In this case the $\mu_{\varepsilon}$ 's are independent. The Grubb measure is the translation invariant intrinsic topological measure for which all the $\mu_{\varepsilon}$ 's are restrictions of the Haar measure. The Grubb measure is symplectic. See [1].

2000 Mathematics Subject Classification: Primary 28C15; Secondary 46G15, 53D25.

Key words and phrases: topological measure, simple measure, Grubb measure, center of mass topological measure. 
Entov and Polterovich ask if this is the only symplectic measure on the torus. During the investigation of this hypothesis we stumbled over the center of mass topological measures. These are simple intrinsic topological measures. From the homotopy formula for the Lie derivative of a differential form, also called Cartan's magic formula, it follows that many of these measures are symplectic.

The technique for constructing the center of mass topological measures is to restrict the above measures to simple smooth closed curves on the torus.

Intrinsic simple topological measures on the torus. Intrinsic topological measures on the torus are determined by their restriction to the solid 2-sided closed sets $\mathcal{C}_{s 2}$. See [2, Definition 8].

Proposition 2. Let $\mathcal{M}$ be the subset of $\mathcal{C}_{s 2}$ consisting of smooth 1-dimensional manifolds $C$, and let $\mu: \mathcal{M} \rightarrow\{0,1\}$ be a function. Then $\mu$ is the restriction of a unique intrinsic simple topological measure if and only if it has the following two properties.

M1 If $C_{1}$ and $C_{2}$ are in $\mathcal{M}$ and $\mu\left(C_{1}\right)=\mu\left(C_{2}\right)=1$, then $C_{1} \cap C_{2} \neq \emptyset$. M2 If $\left\{C_{i}\right\}_{i=1}^{n}$ is a disjoint family of members of $\mathcal{M}$, and $\mu\left(C_{i}\right)=0$ for $1 \leq i \leq n$, then there is a $C \in \mathcal{M}$ disjoint from every $C_{i}$ and with $\mu(C)=1$.

Proof. The "only if" part follows because given disjoint sets $\left\{C_{i}\right\}_{i=1}^{n}$ there is, up to orientation, a unique, surjective function $f: \mathbb{T}^{2} \rightarrow S^{1}$ with $f\left(C_{k}\right)=\exp (2 k \pi i / n)$, and such that $f$ is harmonic in $\mathbb{T}^{2} \backslash \bigcup_{i=1}^{n} C_{i}$. The fibers of $f$ are then all in $\mathcal{M}$, and exactly one of them has measure 1 , since every simple topological measure on the circle $S^{1}$ is a point measure. To prove the converse note that if $\mu$ is the restriction of an intrinsic simple topological measure, then we would have $\mu(D)=1$ for a closed solid 2-sided set $D$ if and only if for every $C \in \mathcal{M}$ with $\mu(C)=1, C \cap D \neq \emptyset$. If we define $\mu$ in this way on closed solid 2-sided sets, the same argument as above shows that the three conditions in [2, Proposition 3] are satisfied.

To each $C \in \mathcal{C}_{s 2}$, there is associated a submodule $L_{C} \in H^{1}\left(\mathbb{T}^{2}, \mathbb{Z}\right)$ of rank one. In fact if we realize $H^{1}\left(\mathbb{T}^{2}, \mathbb{Z}\right)$ as harmonic 1-forms, then $\omega \in L_{C}$ if and only if $\int_{\gamma} \omega=0$ for all closed curves in $\mathbb{T}^{2}$ disjoint from $C$. The function $L: \mathcal{C}_{s 2} \rightarrow\left\{M \mid M\right.$ is a rank one isotropic submodule of $\left.H^{1}\left(\mathbb{T}^{2}, \mathbb{Z}\right)\right\}$ determines an equivalence relation on $\mathcal{C}_{s 2}$ whose partition $\mathcal{E}$ coincides with the one defined in [2, Section 4].

We consider the torus as the quotient $\mathbb{C} / \mathbb{Z}^{2}$. The harmonic differentials $d x$ and $d y$ form a basis for the module $H^{1}\left(\mathbb{T}^{2}, \mathbb{Z}\right)$. The rank one isotropic submodules are in one-to-one correspondence with $\mathbb{Q} \cup\{\infty\}$ as follows: $\alpha(M)=\varepsilon$ 
if and only if for all $\omega=r d x+s d y \in M, \varepsilon=r / s$. The fiber of the function $L$ corresponding to a module $M$ with $\alpha(M)=\varepsilon$ is denoted by $\mathcal{C}_{s 2, \varepsilon}$.

If $C_{1}$ and $C_{2}$ are in $\mathcal{M}$ and $L\left(C_{1}\right) \neq L\left(C_{2}\right)$, then $C_{1} \cap C_{2} \neq \emptyset$. For this reason, the conditions M1 and M2 of Proposition 2 will be satisfied if they are satisfied independently on each equivalence class.

Definition 3. For each $\varepsilon \in \mathbb{Q} \cup\{\infty\}$ we define $\mathcal{M}_{\varepsilon}=\left\{C \mid \alpha\left(L_{C}\right)=\varepsilon\right\}$.

Theorem 4. Let $\mu: \mathcal{M} \rightarrow\{0,1\}$ be a function, and let $\mu_{\varepsilon}: \mathcal{M}_{\varepsilon} \rightarrow$ $\{0,1\}$ be its restriction. Then $\mu$ is the restriction of a unique intrinsic simple topological measure if and only if for each $\varepsilon \in \mathbb{Q} \cup\{\infty\}$ it has the following properties.

M1 If $C_{1}$ and $C_{2}$ are in $\mathcal{M}_{\varepsilon}$ and $\mu\left(C_{1}\right)=\mu\left(C_{2}\right)=1$, then $C_{1} \cap C_{2} \neq \emptyset$. $\mathrm{M} 2$ If $\left\{C_{i}\right\}_{i=1}^{n}$ is a disjoint family of members of $\mathcal{M}_{\varepsilon}$, and $\mu\left(C_{i}\right)=0$ for $1 \leq i \leq n$, then there is a $C \in \mathcal{M}_{\varepsilon}$ disjoint from every $C_{i}$ and with $\mu(C)=1$.

Proof. This is [2, Theorem 32] or [4, Proposition 5.3].

Definition 5. With notation as in the theorem above, we write

$$
\mu=\prod_{\varepsilon \in \mathbb{Q} \cup\{\infty\}} \mu_{\varepsilon}
$$

for the uniquely defined intrinsic simple topological measure $\mu$ on $\mathbb{T}^{2}$ which restricts to all the $\mu_{\varepsilon}$ 's.

On a space which is simply co-connected [4] or of Aarnes genus zero [6], there is a standard procedure for constructing simple topological measures. These measures are the generalized point-measures. On spaces $X$ with $g(X)$ $=0$, the generalized point-measures form a dense subset of the space of all simple topological measures [4]. For general spaces the only known topological measures are, in a sense which is made more precise in the next definition, push-forwards of measures on simpler topological spaces.

DEFINITION 6. If $Y$ is a space and $\mu$ is a topological measure of the form $\mu=f_{*}(\nu)$, where $f: X \rightarrow Y$ is a continuous map and $X$ is a space with $g(X)=0$, we call $\mu$ a patched-on topological measure.

All previously known intrinsic simple topological measures on the torus are of the form $\mu=\prod_{\varepsilon \in \mathbb{Q} \cup\{\infty\}} \mu_{\varepsilon}$, where each restriction $\mu_{\varepsilon}$ is the restriction of a patched-on simple topological measure.

Center of mass topological measures on the torus. In this section we construct the center of mass topological measures, by specifying functions $\mu_{\varepsilon}$ for each $\varepsilon \in \mathbb{Q} \cup\{\infty\}$. In order to do this we need some concepts associated with such an $\varepsilon$. 
Definition 7. A commutative diagram

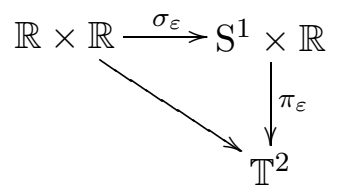

given by

$$
\begin{aligned}
p_{1} \sigma_{\varepsilon}(x, y) & =\bar{x}_{\varepsilon} \equiv p x+q y(\bmod 1), \\
p_{2} \sigma_{\varepsilon}(x, y) & =y_{\varepsilon}=r x+s y, \\
\pi_{\varepsilon}\left(\bar{x}_{\varepsilon}, y_{\varepsilon}\right) & =(\bar{x}, \bar{y})
\end{aligned}
$$

will be called an $\varepsilon$-marking if $\left(\begin{array}{ll}p & q \\ r & s\end{array}\right) \in \mathrm{SL}(2, \mathbb{Z})$ and $\varepsilon=r / s$.

LEMMA 8. If $C \in \mathcal{M}_{\varepsilon}$ and $\pi_{\varepsilon}$ is an $\varepsilon$-marking, then for any parameterization $\gamma: \mathrm{S}^{1} \rightarrow \mathbb{T}^{2}$ of $C$,

$$
\int_{\gamma} d \bar{y}_{\varepsilon}=\int_{\gamma}(r d x+s d y)=0 \quad \text { and } \quad \int_{\gamma} d \bar{x}_{\varepsilon}=\int_{\gamma}(p d x+q d y)= \pm 1 .
$$

Proof. The first integral vanishes by definition of $\mathcal{M}_{\varepsilon}$. Since $d \bar{x}_{\varepsilon}$ and $d \bar{y}_{\varepsilon}$ generate $H^{1}\left(\mathbb{T}^{2}, \mathbb{Z}\right)$ it follows by duality that there is a map $\delta: \mathrm{S}^{1} \rightarrow \mathbb{T}^{2}$ such that $\int_{\delta} d \bar{y}_{\varepsilon}=0$ and $\int_{\delta} d \bar{x}_{\varepsilon}=1$. Hence there is an integer $n$ and a homotopy $\gamma \sim n \delta$. But $C$ is solid so there is an $\eta$ with $\eta \cap \gamma=1$, and hence $n= \pm 1$.

DEFinition 9. With notation as in Lemma 8, we say that a parameterization $\gamma: \mathrm{S}^{1} \rightarrow \mathbb{T}^{2}$ of $C$ is $\pi_{\varepsilon}$-oriented if $\int_{\gamma} d \bar{x}_{\varepsilon}=1$.

LEMMA 10. Let $\pi_{\varepsilon}$ be an $\varepsilon$-marking, $f_{\varepsilon}$ a non-negative function on $\mathrm{S}^{1}$ with $\int_{\mathrm{S}^{1}} f_{\varepsilon}(x) d x=1$, and let $C \in \mathcal{M}_{\varepsilon}$. If we choose a lifting $\widetilde{\gamma}$ of $a \pi_{\varepsilon^{-}}$ oriented parameterization of $C$

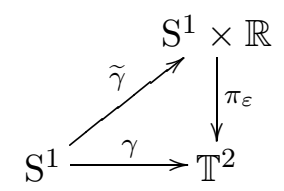

then

$$
\int_{\widetilde{\gamma}} f_{\varepsilon}\left(\bar{x}_{\varepsilon}\right) d \bar{x}_{\varepsilon}=1
$$

and

$$
\int_{\widetilde{\gamma}} y_{\varepsilon} f_{\varepsilon}\left(\bar{x}_{\varepsilon}\right) d \bar{x}_{\varepsilon}
$$

is well defined up to an integer and does not depend on the particular parameterization or the lifting. 
Proof. Let $\delta=\left(1_{\mathrm{S}^{1}}, r\right)$ for some constant $r \in \mathbb{R}$. Since $\gamma$ is a $\pi_{\varepsilon^{-} \text {-oriented }}$ parameterization of $C$, the paths $\widetilde{\gamma}$ and $\delta$ are homotopic. By the Stokes Theorem, since $d\left(f_{\varepsilon}\left(\bar{x}_{\varepsilon}\right) d \bar{x}_{\varepsilon}\right)=0$, we have $\int_{\tilde{\gamma}} f_{\varepsilon}\left(\bar{x}_{\varepsilon}\right) d \bar{x}_{\varepsilon}=\int_{\delta} f_{\varepsilon}\left(\bar{x}_{\varepsilon}\right) d \bar{x}_{\varepsilon}$, and the first statement follows by the definition of $f_{\varepsilon}$. The second statement follows since any two liftings differ by an integer.

DeFinition 11. Given an $\varepsilon$-marking $\pi_{\varepsilon}$, a real number $0 \leq r_{\varepsilon}<1$, and a distribution-function $f_{\varepsilon}$, the function $\mu_{\varepsilon}=\mu\left(\pi_{\varepsilon}, f_{\varepsilon}, r_{\varepsilon}\right): \mathcal{M}_{\varepsilon} \rightarrow\{0,1\}$ is defined by the formula

$$
\mu_{\varepsilon}(C)=1 \quad \text { if and only if } \int_{\widetilde{\gamma}} y_{\varepsilon} f_{\varepsilon}\left(\bar{x}_{\varepsilon}\right) d \bar{x}_{\varepsilon} \equiv r_{\varepsilon}(\bmod 1),
$$

where $\widetilde{\gamma}$ is a lifting of a $\pi_{\varepsilon}$-oriented parameterization of $C$.

Theorem 12. If for each $\varepsilon \in \mathbb{Q} \cup\{\infty\},\left(\pi_{\varepsilon}, f_{\varepsilon}, r_{\varepsilon}\right)$ is a triple as in Definition 11, then there is a unique intrinsic simple topological measure $\mu$ on $\mathbb{T}^{2}$ restricting to the function $\mu_{\varepsilon}=\mu\left(\pi_{\varepsilon}, f_{\varepsilon}, r_{\varepsilon}\right)$ on each $\mathcal{M}_{\varepsilon}$. We call $\mu$ the center of mass topological measure associated to the triples $\left(\pi_{\varepsilon}, f_{\varepsilon}, r_{\varepsilon}\right)$.

Proof. We have to show that for any $\varepsilon$, the function $\mu_{\varepsilon}$ satisfies the conditions M1 and M2 of Theorem 4. Let $C_{1}$ and $C_{2}$ be disjoint members of $\mathcal{M}_{\varepsilon}$, and let $\widetilde{\gamma}_{1}$ and $\widetilde{\gamma}_{2}$ be liftings of $\pi_{\varepsilon}$-oriented parameterizations of $C_{1}$ and $C_{2}$. We may choose the liftings such that $0<p_{2} \circ \widetilde{\gamma}_{2}(0)-p_{2} \circ \widetilde{\gamma}_{1}(0)<1$. By the Stokes Theorem, the difference

$$
r=\int_{\widetilde{\gamma}_{2}} y_{\varepsilon} f_{\varepsilon}\left(\bar{x}_{\varepsilon}\right) d \bar{x}_{\varepsilon}-\int_{\widetilde{\gamma}_{1}} y_{\varepsilon} f_{\varepsilon}\left(\bar{x}_{\varepsilon}\right) d \bar{x}_{\varepsilon}=\int_{D} f_{\varepsilon}\left(\bar{x}_{\varepsilon}\right) d \bar{x}_{\varepsilon} \wedge d y_{\varepsilon}
$$

is a weighted area between the curves, and since the curves are disjoint we have $0<r<1$. This proves M1. To prove M2 we may use the same technique as in the "only if" part of the prof of M2 in Proposition 2.

REMARK 13. This construction has recently been generalized by Grubb to all spaces of Aarnes genus one. See [3].

REMARK 14. To see that a center of mass topological measure is not patched-on, notice that for any real number $R>0$, there is a smooth surjective function $f: \mathbb{T}^{2} \rightarrow S^{1}$ such that no connected component of any lifting to $\mathrm{S}^{1} \times \mathbb{R}$ of any fiber $f^{-1}(\exp (i t))$ is contained in $\mathrm{S}^{1} \times[-R, R]$. Just consider a function whose fibers are sufficiently tilted meridians. That could be $f(\bar{x}, \bar{y})=e^{2 \pi i(\bar{y}-R \sin 2 \pi \bar{x}}$. For the center of mass topological measure associated to the uniform distribution function, we can find arbitrarily tilted meridians with measure 1 .

THEOREM 15. A center of mass topological measure associated with a family of triples $\left(\pi_{\varepsilon}, 1, r_{\varepsilon}\right)$, meaning that all the distributions are uniform, is a symplectic topological measure in the sense of [1]. 
Proof. Since the torus is a surface, all topological measures give rise to strongly quasi-linear functionals. Furthermore the intrinsic topological measures vanish on all displaceable sets. Hence it remains only to show invariance under symplectomorphisms homotopic to the identity. It suffices to do this infinitesimally, so let $f$ be a smooth function on the torus and let $X$ be the corresponding Hamiltonian vector field. This means that $X\lrcorner$ $(d \bar{x} \wedge d \bar{y})=X\lrcorner\left(d \bar{x}_{\varepsilon} \wedge d \bar{y}_{\varepsilon}\right)=d f$. Here we have used the sign convention of Bourbaki, Algèbre, Chapitre 3, where the left interior multiplication of a vector and an exterior form is defined by the rule $\langle Z, X\lrcorner \omega\rangle=\langle Z \wedge X, \omega\rangle$. For any vector $X$, the operator $i(X): \bigwedge T^{*} \rightarrow \bigwedge T^{*}$ defined on $p$-forms by $\left.i(X)(\omega)=(-1)^{p-1} X\right\lrcorner \omega$ is an anti-derivation of degree -1 , and the Lie derivative of differential forms is give by the celebrated formula

$$
\mathcal{L}_{X}=i(X) \circ d+d \circ i(X)
$$

Let $C \in \mathcal{M}_{\varepsilon}$, and let $\widetilde{\gamma}$ be a lifting of a $\pi_{\varepsilon}$-oriented parameterization of $C$. We also let $\tilde{f}$ and $\widetilde{X}$ be liftings of $f$ and $X$. If $C_{t}$ denotes the image of the set $C$ after having drifted with the flow of $X$ for a time $t$, and $\widetilde{\gamma}_{t}$ is its lifting, then

$$
\mu_{\varepsilon}\left(C_{t}\right)=1 \quad \text { if and only if } \int_{\tilde{\gamma}_{t}} y_{\varepsilon} d \bar{x}_{\varepsilon} \equiv r_{\varepsilon}(\bmod 1) .
$$

From the homotopy formula we have

$$
\begin{aligned}
\frac{d}{d t}\left(\int_{\widetilde{\gamma}_{t}} y_{\varepsilon} d \bar{x}_{\varepsilon}\right) & \left.\left.=\int_{\widetilde{\gamma}} \mathcal{L}_{\widetilde{X}}\left(y_{\varepsilon} d \bar{x}_{\varepsilon}\right)=\int_{\widetilde{\gamma}}\left(d(\widetilde{X}\lrcorner\left(y_{\varepsilon} d \bar{x}_{\varepsilon}\right)\right)-\widetilde{X}\right\lrcorner\left(d y_{\varepsilon} \wedge d \bar{x}_{\varepsilon}\right)\right) \\
& \left.=\int_{\widetilde{\gamma}}\left(d(\widetilde{X}\lrcorner\left(y_{\varepsilon} d \bar{x}_{\varepsilon}\right)\right)+d \widetilde{f}\right)=0,
\end{aligned}
$$

and therefore the value $\mu_{\varepsilon}\left(C_{t}\right)$ remains constant.

REMARK 16. It is possible to prove that the only intrinsic, translation invariant symplectic measure is the Grubb measure, but the proof I know is very long and highly technical and will not be included.

\section{References}

[1] M. Entov and L. Polterovich, Quasi-states and symplectic intersections, to appear.

[2] D. J. Grubb, Irreducible partitions and the construction of quasi-measures, Trans. Amer. Math. Soc. 353 (2001), 2059-2072.

[3] - Covering spaces and topological measures, to appear.

[4] F. F. Knudsen, Topology and the construction of extreme quasi-measures, Adv. Math. 120 (1996), 302-321.

[5] J. Aarnes, Quasi-states and quasi-measures, ibid. 86 (1991), 41-67. 
[6] J. Aarnes, Construction of non-subadditive measures and discretization of Borel measures, Fund. Math. 147 (1995), 213-237.

Department of Mathematical Sciences

Norwegian University of Science and Technology

NO-7491, Trondheim, Norway

E-mail: knudsen@math.ntnu.no

Received 14 February 2005 